\title{
Case Report of Neuron-Binding IgGs in ALS Patient Serum in Argentina
}

\author{
Giuliana C. Di Mauro', Bruno De Ambrosi², Osvaldo D. Uchitel ${ }^{1,2}$ and Graciela L. Mazzone ${ }^{2,3 *}$ \\ ${ }^{1}$ Instituto de Fisiología, Biología molecular y Neurociencias, UBA CONICET, Departamento de Fisiología, Biología Molecular y Celular, Facultad de \\ Ciencias Exactas y Naturales, Universidad de Buenos Aires, Argentina \\ ${ }^{2}$ Fundación para la Lucha contra las Enfermedades Neurológicas de la Infancia (FLENI), Buenos Aires, Argentina \\ ${ }^{3}$ Instituto de Investigaciones en Medicina Traslacional (IIMT), CONICET-Universidad Austral, Derqui-Pilar, Buenos Aires, Argentina
}

${ }^{*}$ Corresponding author: Graciela L. Mazzone, Instituto de Medicina Traslacional (IIMT), CONICET-Universidad Austral, Av. Juan Domingo Perón 1500, B1629AHJ Pilar, Argentina; Tel: +54 230 4387425; E-mail: GMazzone@austral.edu.ar

Received: June 18, 2021; Accepted: June 28, 2021; Published: July 15, 2021

\begin{abstract}
Amyotrophic Lateral Sclerosis (ALS) is a degenerative disorder characterized by ongoing loss of motoneurons. The etiology of the sporadic ALS form it is thought to be related to immune-mediated motoneuron degeneration and death. The present study was designed to describe the effect of serum factors derived from sporadic ALS patients on mouse spinal cord preparations in vitro. Sera from patients with sporadic ALS were collected and used for immunofluorescence analysis to investigate their effects on neuronal survival and microgliosis. Our experiments demonstrated that $5 \mathrm{~h}$ application of serum factors (derived from three ALS patients) labeled with human IgG secondary antibody were localized to ventral spinal neurons identified with the NeuN marker. Moreover, a significant reduction in the number of ventral NeuN positive cells was observed with serum from a patient who suffered from upper motor neuron signs as criteria for ALS diagnosis (20\% less compared to sham, spinal cord preparations without serum). No change in microglia number was found after exposure to ALS sera, although in two cases a significant decrease in microglial branch length was observed (28-32\%). Microglia morphology showed increased number of end points and branches with serum from the patient with upper motor neuron symptoms. These observations were absent after control sera. Our data indicate that spinal cord cultures can be a useful model to further characterize the pathological processes of sporadic ALS and the immune mechanism as previously suggested in vivo. Future studies are needed to unveil the molecular mechanisms underlying this preferential targeting of neurons and microglia by ALS serum.
\end{abstract}

Keywords: Amyotrophic lateral sclerosis, Autoimmunity, Microglial activation, Neuron labeling

\section{Non-standard Abbreviations}

ALS, Amyotrophic Lateral Sclerosis; HC1 (2), serum form healthy control 1 (2); Iba-1, ionized calcium-binding adapter molecule; IgG, immunoglobulin; MNs, motor neurons; NeuN, neuronal-specific nuclear protein; P1 (2 and 3) serum from ALS sporadic patient 1 (2 and 3); PBS, phosphate-buffered saline.

\section{Highlights}

- Immune mechanisms contribute to ALS pathology at spinal cord.

- Unlike controls, ALS serum factors bound spinal ventral neurons.

- Microglia morphological changes were induced by ALS serum factors.

- In vitro spinal preparations can be a useful platform to study immunopathology of ALS.

\section{Introduction}

Amyotrophic Lateral Sclerosis (ALS) is a neurodegenerative disease that affects motoneurons with fatal outcome usually within $2-5$ years [1].
Although the aetiology and pathogenesis of ALS are unknown increasing evidence supports immune-mediated mechanisms, although this remains controversial [2]. Our previous studies have demonstrated that motor nerve terminals are a target for autoimmune response, induced by immunoglobulin's (IgGs) from sporadic ALS patients, resulting in neuromuscular dysfunction in vivo [3]. Indeed, IgGs obtained from patients with ALS reduce $\mathrm{Ca}^{2+}$ transients and glutamate receptor desensitization so that excitotoxic damage is facilitated in a culture brain neuron model [4]. Furthermore, we have shown that subcutaneous injection of ALS-IgG into the levator auris muscle were immunoreactive to spinal motoneurons, preferentially accumulate in the soma by binding to unknown specific cytoplasmic targets [5]. The question then arises about the role of ALS serum factors on spinal cord neurons and the inflammatory process that would be associated with the disease onset and development. In vitro spinal cord preparations have been exploited as a model to investigate early pathophysiological mechanisms of spinal cord damage and developmental changes in cellular profiles [6]. The aim of the present study was to investigate, using neonatal mouse spinal cord preparations in vitro, the effect of serum from sporadic ALS patients. Thus, sera from patients with sporadic ALS diagnosis in accordance with the El Escorial criteria [7] and from healthy volunteer subjects were tested on neuronal numbers and microglia morphology. 


\section{Materials and Methods}

\section{Preparation of Mouse Spinal Cord Cultures and ALS Serum Incubation}

Thoracolumbar spinal cord preparations were isolated from neonatal C57BL/6 mice (1-3 day old) in accordance with standard procedures [8]. Briefly, spinal cords were dissected with Krebs' solution of the following composition (in $\mathrm{mM}$ ): $\mathrm{NaCl}, 113 ; \mathrm{KCl}, 4.5$; $\mathrm{MgCl}_{2} .7 \mathrm{H}_{2} \mathrm{O}, 1 ; \mathrm{CaCl}_{2}, 2 ; \mathrm{NaH}_{2} \mathrm{PO}_{4}, 1 ; \mathrm{NaHCO}_{3}, 25$; glucose, 11 ; gassed with $95 \% \mathrm{O} 25 \% \mathrm{CO}_{2} ; \mathrm{pH} 7.4$ at room temperature. After dissection spinal slice cultures were incubated with sera from sporadic ALS patients (listed as P1, P2 and P3) in standard Krebs' solution (1:1000) for $5 \mathrm{~h}$ at room temperature. Patients were diagnosed with sporadic ALS according to clinical criteria that included progressive paralysis with mixed upper and lower motor neuron signs (1 woman and 2 men) [7]. Healthy control serum was collected from two subjects ( $\mathrm{HC} 1$ and $\mathrm{HC} 2$ ) with no evidence of neurological disease. The serum samples were obtained with prior consent from patients attending the FLENI clinic. Mouse spinal cord controls, without any serum treatment (sham), were processed in parallel to test preparations in each experiment. After incubation, all spinal cord tissues were immediately fixed in phosphate-buffered saline (PBS) containing $4 \%$ paraformaldehyde $\left(24 \mathrm{~h}\right.$ at $\left.4^{\circ} \mathrm{C}\right)$ followed by $30 \%$ sucrose $\mathrm{PBS}$ for cryoprotection $\left(24 \mathrm{~h}\right.$ at $\left.4^{\circ} \mathrm{C}\right)$ for immunostaining.

\section{Slice Immunostaining and Cell Analysis}

Full details of this procedure have previously been published [6]. In brief, spinal cord slices (30- $\mu \mathrm{m}$ thick) were collected sequentially on histology slides. Slices were blocked with fetal calf serum (3\%), bovine serum albumin (3\%) and Triton X-100 (0.3\%) in PBS for $1 \mathrm{~h}$ at room temperature, followed by overnight incubation at $4^{\circ} \mathrm{C}$ in blocking solution containing the following antibodies: NeuN (neuronalspecific nuclear protein, 1:300, Millipore, Billerica, MA, USA) for neurons and Iba-1 (ionized calcium-binding adapter molecule 1, 1:500, Wako, Osaka, Japan) for microglia. Primary antibodies were visualized using the corresponding secondary fluorescent antibody (at 1:500 dilution; Invitrogen, Carlsbad, CA, USA). The serum factors from ALS patients were recognized by an anti-human IgG secondary antibody (at 1:500 dilution; Invitrogen). Since the staining was diffuse, data quantification was performed in terms of immunofluorescence intensity (expressed in arbitrary units, AU) obtained with a line scan of each image to verify the nuclear distribution of IgG according to NeuN soma size measured with ImageJ software (NIH, https://imagej. nih.gov/ij/index.html). For each slice culture, the number of NeuN positive cells was obtained by counting stacks of 10 images (40x magnification) with FV300 confocal microscope (Olympus Optical, Tokyo, Japan), and quantified using ImageJ software. NeuN positive cells were counted in two ROIs (namely, dorsal, and ventral) in an area $=90488,5 \mu \mathrm{m}^{2}$. In view of the very large number of histological sections provided by each spinal cord the final numbers of counted cells were expressed as fold average respect to sham, obtained from an equivalent number of sections by experiment.

The morphology of Ibal-positive cells was analysed by the 'skeleton' method [9]. Thus, the signal from Iba1-positive processes was enhanced to optimize their detection followed by noise despeckling to eliminate background fluorescence. The resulting images were converted to binary data and then 'skeletonized' by using ImageJ software. The Analyze Skeleton plug-in (http://imagejdocu.tudor. lu) was then applied to Iba- 1 images to collect mean raw data on the number of branches, end points and process lengths.

\section{Data Analysis}

Data were expressed as means \pm S.E.M; $\mathrm{n}=$ number of slices from 5 different independent experiments. Statistical analysis was carried out with SigmaStat (SigmaStat 3.1, Systat Software, Chicago, IL, USA). For multiple comparisons, the analysis of variance (ANOVA) test for parametric data followed by the Tukey-Kramer post hoc test was used. Nonparametric values were analyzed with the Kruskal-Wallis test. The accepted level of significance was always $\mathrm{p}<0.05$.

\section{Results}

Clinical diagnosis of sporadic ALS was achieved by careful patient history collection, plus physical and neurological examination according to El Escorial criteria [7] and evidence of signs of lower or upper motor neuron degeneration. Hence, two patients were classified with lower motor neuron (P1 and P3) and one patient with upper motor neuron degeneration (P2). In vitro slice preparations of the neonatal spinal cord were treated with healthy control serum $(\mathrm{HC})$, patient serum $(\mathrm{P})$ or without serum (sham condition) for $5 \mathrm{~h}$ at room temperature. Figure 1A shows examples of immunohistochemistry analysis to identify neurons (NeuN, in green. Figure 1A top row), human immunoglobulins (IgG, light blue, Figure 1A lower row) and microglia (Iba-1, in red, Figure 1E).

\section{Ventral Neuronal Labeling with ALS Serum Factors}

Figure $1 \mathrm{~A}$ and $1 \mathrm{~B}$ shows that serum factors from all ALS patients labeled with the anti-IgG human secondary antibody were localized to NeuN positive neuronal cells. The quantification of mean immunofluorescence intensity for IgG signal was analyzed for different neuronal soma size. Figure 1B demonstrates higher mean fluorescence intensity (arbitrary units, $\mathrm{AU}$ ) for P1, P2 and P3 in comparison to sham and $\mathrm{HC} 1$ and $\mathrm{HC} 2$ in the ventral spinal region. On the contrary a unique soma size IgG signal labeling was observed at dorsal region, data not shown. As exemplified in Figure 1C and 1D, the number of dorsal NeuN positive cells was consistent throughout samples. Conversely, a significant reduction in neuron number at the ventral spinal region was observed following serum from patient $2\left({ }^{* *} \mathrm{p} \leq 0.01\right.$ vs. sham, $\mathrm{n}=3-10$, Kruskal-Wallis one-way analysis of variance on ranks test).

\section{Microglial Phenotypes Observed after ALS Serum Factors}

The immunohistochemical staining for Iba-1 microglia marker was evaluated in ventral spinal regions after ALS serum incubation, as shown in Figure 1E. There was no significant change in the number of Iba1-positive cells. However, a morphological change in microglia was observed for P2 with significant increase in the number of microglial branches and endpoints (Figure $1 \mathrm{~F}$ and $1 \mathrm{G},{ }^{* * *} \mathrm{p} \leq 0.001$ vs. sham, $\& \& \& \mathrm{p} \leq 0.001$ vs. HC, $\mathrm{n}=4-10$, Mann-Whitney test). The vast majority microglia of $\mathrm{P} 1$ and $\mathrm{P} 2$ also showed a significant reduction in branch length (Figure $1 \mathrm{H},{ }^{*} \mathrm{p} \leq 0.05$ vs. sham, $\mathrm{n}=4-10$, Mann-Whitney test). 
A NeuN - IgG
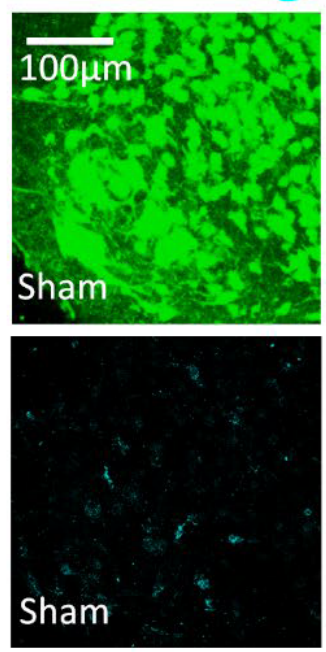

B

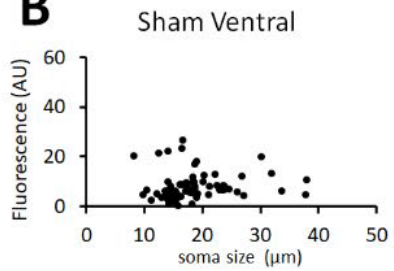

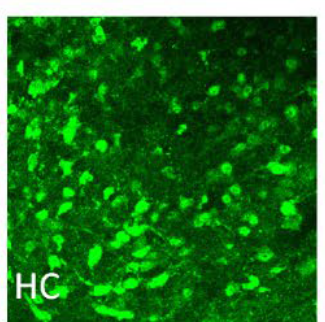

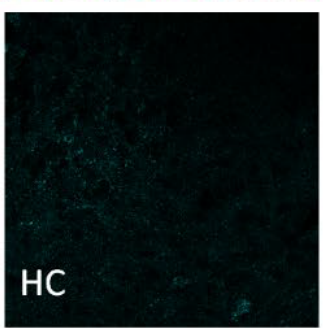

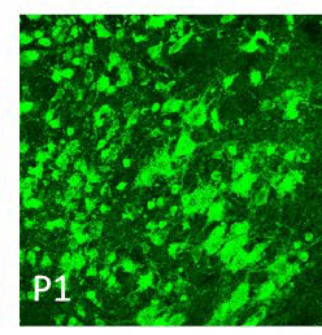

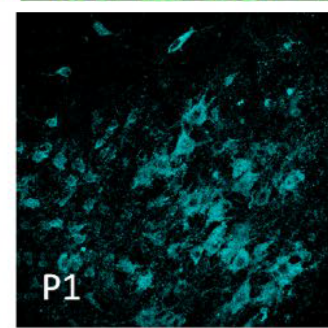

HC Ventral

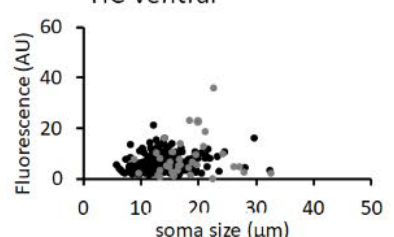

P2 Ventral
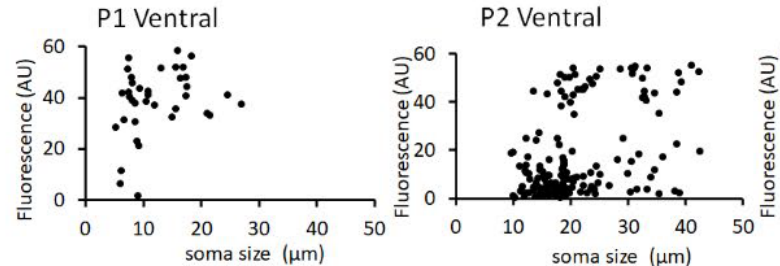

soma size $(\mu \mathrm{m})$
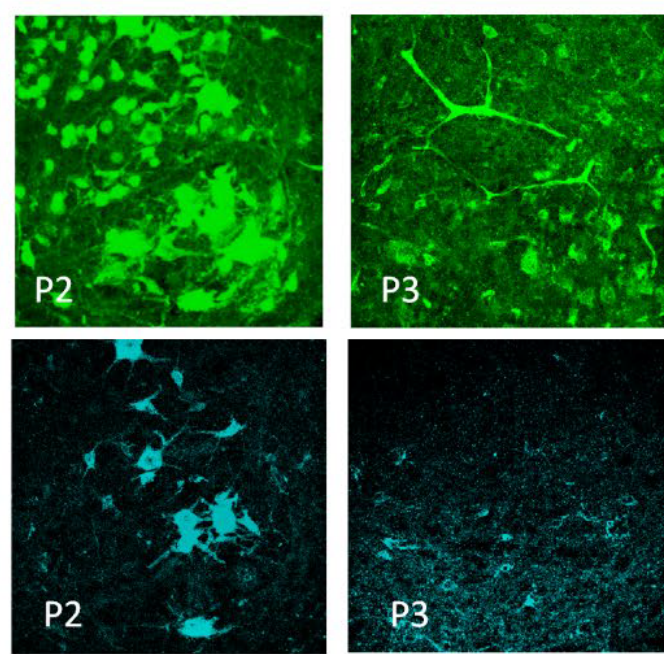

$\mathrm{C} \cong$

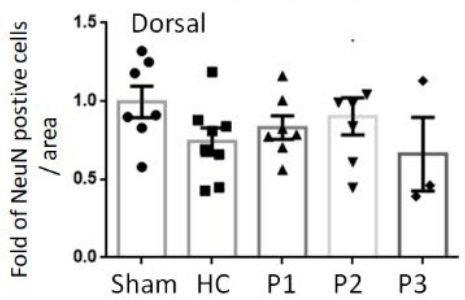

P3 Ventral

\section{E Iba-1}
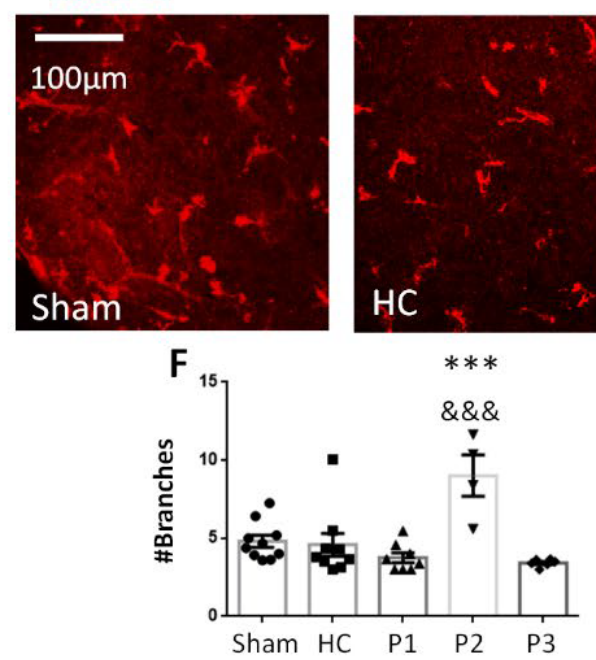

Sham HC P1 P2 P3

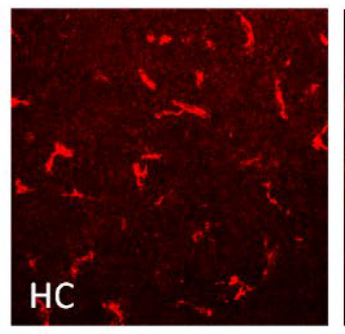

G

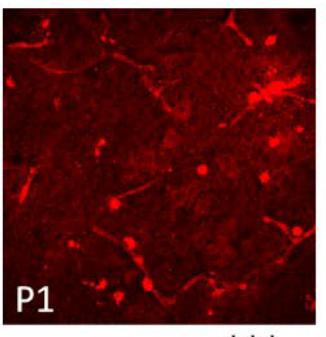

$* * *$
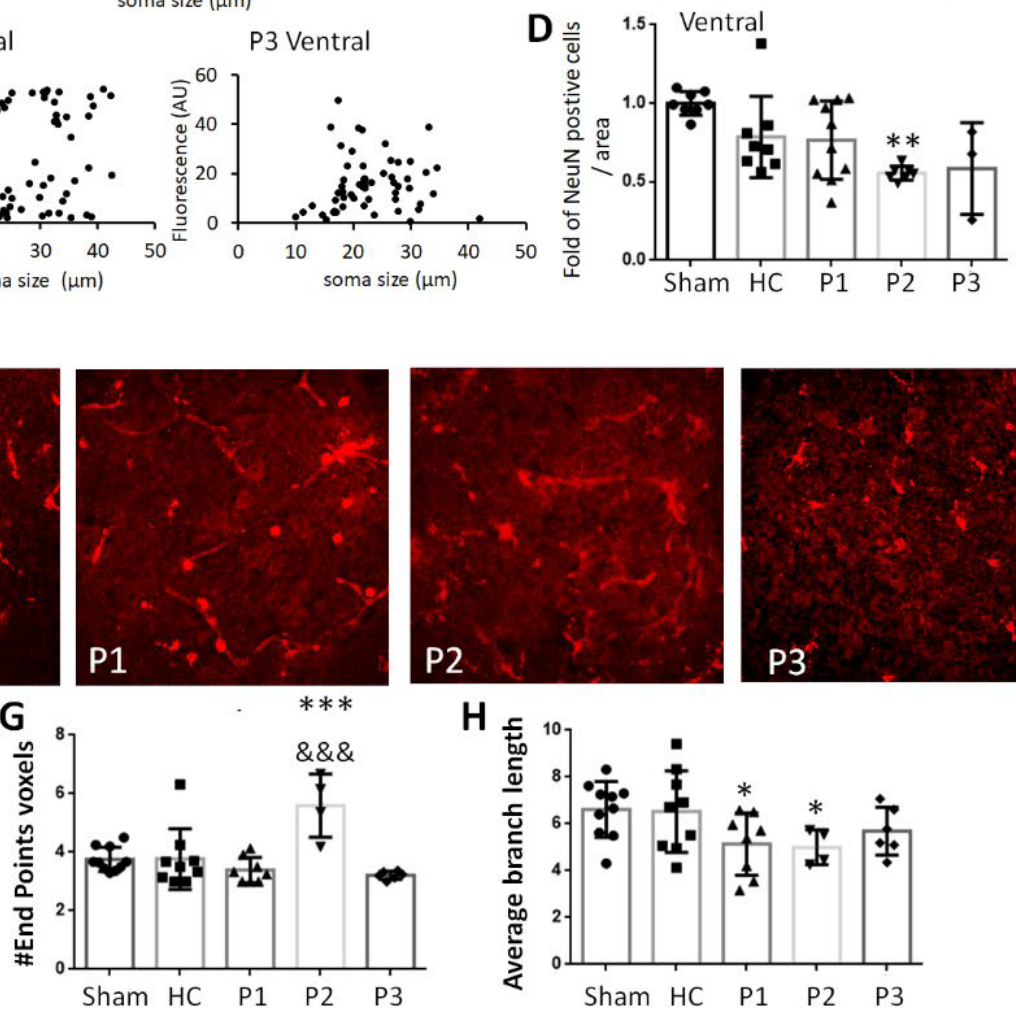
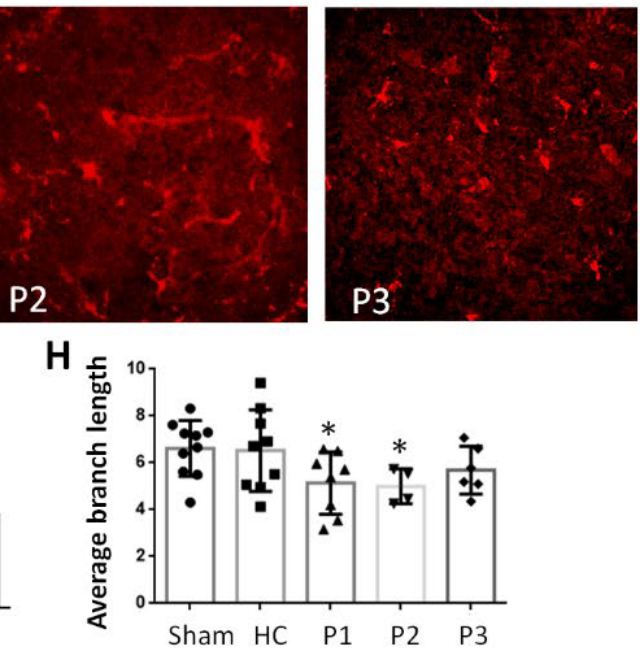

Figure 1: Neurons and microglia immunolabeling after incubation with serum factors derived from ALS patients in vitro spinal cord preparations. (A) Examples of neuron staining (NeuN, green) and serum factors that were identified by anti-human IgG labeling (light blue) in the ventral region of the spinal cord for sham, healthy serum (HC), serum from patients with sporadic ALS (P1, P2 and P3). (B) Plots showing IgG mean fluorescence intensity quantification (arbitrary units, AU) that colocalized with NeuN staining by measuring different neuronal soma size $(\mu \mathrm{m})$. Results are expressed as raw data for in vitro spinal cord preparations for sham, HC (HC1 grey dots and HC2 black dots, respectively) and for P1, P2 and P3 at ventral region. (C-D) Histograms showing the fold of NeuN positive cells in dorsal and ventral area related to sham condition, or after $5 \mathrm{~h}$ of incubation. No differences in the number of neurons were found in dorsal spinal cord region. (D) There was a significant decrease in the number of neurons following serum from P2 at the ventral region, $\mathrm{n}=3-10$, ${ }^{* *} \mathrm{p}<0.05 \mathrm{vs}$. sham. (E-H) Iba-1 morphological changes after ALS serum incubation. (E) Examples of microglia staining (Iba-1 in red) in mouse isolated spinal cord. Histograms showing the raw data for number of branches (F), end points voxels $(\mathbf{G})$, and average branch length $(\mathbf{H}$, in $\mu \mathrm{m})$ for Iba-1 in ventral region of spinal cord cultures. In the ventral region there was a significant change in microglia morphology for P2, with a

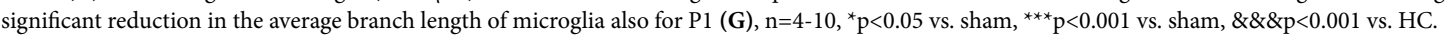




\section{Discussion}

Most studies describe the role ALS effects on lower MNs in the spinal cord and brain stem, and upper MNs in the motor cortex [10]. While ALS is a multifactorial disease with diverse aetiology, the main cause of its onset in the sporadic form is still unknown, although it is suspected that the activity of the immune system impacts its course and development [1]. Several studies show that, in this immune response, the IgG of the patients play a fundamental role as triggers of the disease [2]. The present report shows that serum factors derived from three patients labelled with human IgG secondary antibody were localized to ventral spinal neurons. Interestingly, the sera of all three ALS sporadic patients induced large soma size labeling of ventral neurons and microgliosis in in vitro spinal cord preparations.

\section{Ventral Neurons were Labeled by ALS Serum Factors}

Our previous studies demonstrated that ALS-IgG injected into the mouse levator auris muscle significantly immune react with nerve terminals and, by retrograde axonal transport, are actively accumulated in the MNs soma [5]. Here we have shown that most large soma size ventral spinal neurons were immunoreactive to serum factors derived from patients with the sporadic ALS form. Indeed, we could not observe a clear preference of ALS serum for a certain neuronal type. However, in this study it is expected that ALS IgG have been in contact with every single neuron while, in our previous study, ALS IgG reached the MNs via the motor axons. We have shown that the cellular composition varies between dorsal and ventral spinal regions, the latter being characterized by the presence of differential soma size interneurons as well as MNs [6]. Indeed, our pervious data have also demonstrated that ALS does not affect all neurons, but mainly certain types of MNs [5]. Our early studies have shown that the delayed neuroprotection by riluzole after kainate treatment, to mimic excitotoxicity as one major factor MN degeneration in ALS, was observed in the dorsal and central regions, but not in the ventral one [11]. The different neuronal vulnerability in the spinal cord $[8,12]$, probably related to the neuron-selective ability by different protocols to induce neuroprotection, might highlight preference of ALS serum binding to large ventral neurons. Our results also demonstrate that binding of serum is not a trigger for neurodegeneration, at least within the short time of experimental serum application. A likely explanation, in addition to selective large cell binding, is that MNs have a delicate metabolic state and are perhaps more susceptible to any intracellular signalling process that can be initiated by the pathological ALS serum in vitro [13]. Further studies are needed to clarify the molecules responsible for this phenomenon, as well as the mechanism of IgG to differential cell labeling on sporadic ALS onset.

\section{Microglia Morphological Changes Induced by ALS Serum Factors}

To advance our understanding of ALS pathogenesis and the role of inflammatory processes, an immunofluorescence study with Iba-1, a microglial marker, was performed. Inflammation is mostly related to deterioration in neuronal function and involves a change of microglia number or morphological phenotypic changes. Our results demonstrated that the number of microglia in both dorsal and ventral areas did not vary after incubation with serum from patients or voluntary healthy controls.
A simple explanation for this result is that neuroinflammation (if any) would not be initially characterized by microglia proliferation [14]. However, another important characteristic of neuroinflammation is the different states of microglia activation. When the inflammatory process occurs, microglia can pass from rest to different phenotypic active states [14]. In the present study serum factors derived from a patient with upper motoneuron degeneration induced significant microglia morphological change. Further studies will be needed to determine if this inflammatory change is a cause or consequence of neuronal degeneration.

\section{Final Considerations and Conclusion}

The present findings provide further evidence in favour of immunological mechanisms contributing to ALS pathology and disease progression in the spinal cord. In vitro spinal cord preparation is a useful model to explore the basic molecular mechanisms and cellular consequences of ALS.

\section{Acknowledgments}

We thank Maria Eugenia Martin for the assistance with the spinal preparations. We thank Dr. Andrea Nistri and Dr. Carly Mc Carthy for invaluable comments and critical reading of the manuscript. This study was supported by Universidad Austral, FLENI, CONICET and Grant 01/Q666 (20020130100666BA; Universidad de Buenos Aires Ciencia y Tecnología [UBACYT]) from University of Buenos Aires (to O.D.U.).

\section{Declaration of Competing Interest}

None declared.

\section{References}

1. Beers DR, Appel SH (2019) Immune dysregulation in amyotrophic lateral sclerosis: mechanisms and emerging therapies. Lancet Neurol 18:211-220. [crossref]

2. Pagani MR, Gonzalez LE, Uchitel OD (2011) Autoimmunity in amyotrophic lateral sclerosis: past and present. Neurol Res Int 2011: 497080. [crossref]

3. Gonzalez LE, Kotler ML, Vattino LG, Conti E, Reisin RC, et al. (2011) Amyotrophic lateral sclerosis-immunoglobulins selectively interact with neuromuscular junctions expressing P/Q-type calcium channels. J Neurochem 119: 826-838. [crossref]

4. Andjus PR, Khiroug L, Nistri A, Cherubini E (1996) ALS IgGs suppress [Ca2+]i rise through P/Q-type calcium channels in central neurones in culture. Neuroreport 7: 1914-1916. [crossref]

5. Fratantoni SA, Dubrovsky AL, Uchitel OD (1996) Uptake of immunoglobulin G from amyotrophic lateral sclerosis patients by motor nerve terminals in mice. J Neurol Sci 137:97-102. [crossref]

6. Cifra A, Mazzone GL, Nani F, Nistri A, Mladinic M (2012) Postnatal developmental profile of neurons and glia in motor nuclei of the brainstem and spinal cord, and its comparison with organotypic slice cultures. Dev Neurobiol 72:1140-1160. [crossref]

7. Brooks BR (1994) El Escorial World Federation of Neurology criteria for the diagnosis of amyotrophic lateral sclerosis. Subcommittee on Motor Neuron Diseases/ Amyotrophic Lateral Sclerosis of the World Federation of Neurology Research Group on Neuromuscular Diseases and the El Escorial "Clinical limits of amyotrophic lateral sclerosis" workshop contributors. J Neurol Sci 124: 96-107. [crossref]

8. Mazzone GL, Margaryan G, Kuzhandaivel A, Nasrabady SE, Mladinic M, et al (2010) Kainate-induced delayed onset of excitotoxicity with functional loss unrelated to the extent of neuronal damage in the in vitro spinal cord. Neuroscience 168: 451-462. [crossref]

9. Mazzone GL, Mladinic M, Nistri A (2013) Excitotoxic cell death induces delayed proliferation of endogenous neuroprogenitor cells in organotypic slice cultures of the rat spinal cord. Cell Death Dis 4: e902. https://doi.org/10.1038/cddis.2013.431

10. Shaw PJ (1999) Motor neurone disease. BMJ 318:1118-1121. https://doi.org/10.1136/ bmj.318.7191.1118 
Graciela L. Mazzone (2021) Case Report of Neuron-Binding IgGs in ALS Patient Serum in Argentina

11. Mazzone GL, Nistri A (2011) Delayed neuroprotection by riluzole against excitotoxic damage evoked by kainate on rat organotypic spinal cord cultures. Neuroscience 190: 318-327. [crossref]

12. Taccola G, Margaryan G, Mladinic M, Nistri A (2008) Kainate and metabolic perturbation mimicking spinal injury differentially contribute to early damage of locomotor networks in the in vitro neonatal rat spinal cord. Neuroscience 155: 538555. [crossref]
13. Comoletti D, Muzio V, Capobianco A, Ravizza T, Mennini T (2001) Nitric oxide produced by non-motoneuron cells enhances rat embryonic motoneuron sensitivity to excitotoxins: comparison in mixed neuron/glia or purified cultures. J Neurol Sci 192: 61-69. [crossref]

14. Graeber MB (2010) Changing face of microglia. Science 330: 783-788.

\section{Citation:}

Di Mauro GC, De Ambrosi B, Uchitel OD, Mazzone GL (2021) Case report of neuron-binding IgGs in ALS patient serum in Argentina. J Neurol Neurocrit Care Volume 4(2): 1-5. 